\title{
AUTONOMOUS TRAJECTORY GENERATION FOR MOBILE ROBOTS WITH NON-HOLONOMIC AND STEERING ANGLE CONSTRAINTS*
}

\author{
François G. Pin and Hubert A. Vasseur \\ Center for Engineering Systems Advanced Research \\ Oak Ridge National Laboratory \\ P.O. Box 2008 \\ Building 6025, MS-6364 \\ Oak Ridge, Tennessee 37831-6364
}

CONF-9008122--1

DE90 012298

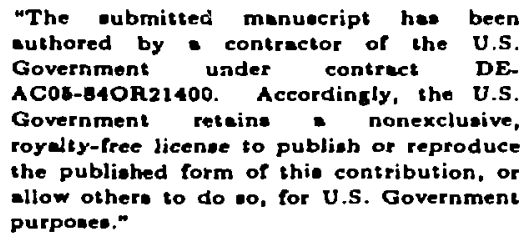

To be published in the Proceedings of the IEEE International Workshop on Intelligent Motion Control, Istanbul, Turkey, August 20-22, 1990.

* Research sponsored by the Engineering Research Program of the Office of Basic Energy Sciences, of the U.S. Department of Energy, under contract No. DE-AC05-84OR21400 with Martin Marietta Energy Systems, Inc., by the Advanced Concept and Technology Program of the Army Material Command under Interagency Agreement No. 1495-CO92-A1 with the U.S. DOE, and by the Fort Belvoir Research Development and Engineering Center under Interagency Agreement No. 0883-CO11-A1 with the U.S. DOE. 


\title{
AUTONOMOUS TRAJECTORY GENERATION FOR MOBILE ROBOTS WITH NON-HOLONOMIC AND STEERING ANGLE CONSTRAINTS
}

\author{
François G. Pin and Hubert A. Vasseur \\ Center for Engineering Systems Advanced Research \\ Oak Ridge National Laboratory \\ P.O. Box 2008 \\ Building 6025, MS-6364 \\ Oak Ridge, Tennessee 37831-6364
}

\begin{abstract}
This paper presents an approach to the trajectory planning of mobile platforms characterized by non-holonomic constraints and constraints on the steering angle and steering angle rate. The approach is based on geometric reasoning and provides deterministic trajectories for all pairs of initial and final configurations (position $x, y$, and orientation $\Theta$ ) of the robot. Furthermore, the method generates trajectories taking into account the forward and reverse mode of motion of the velicle, or combination of these when complex maneuvering is involved or when the environment is obstructed with obstacles. The trajectory planning algorithm is described, and examples of trajectories generated for a variety of environmental conditions are presented. The generation of the trajectories only talies a few milliseconds of run time on a micro Vax, making the approach quite attractive for use as a real-time motion planner for teleoperated or sensor-based autonomous velicles in complex environments.
\end{abstract}

\section{Introduction}

Autonomous mobile robots have raised high hopes in the military and industrial communities because of the potential safety improvement and gain of productivity they may provide. These applications require sturdy three- or four-whecl mobile platforms, the kinematics of which are very similar to that of a car in the sense that steering is performed using one or two front wheels and the steering angle of the wheels is bounded. The fact that the two rear wheels are not steerable and roll without slipping on the ground introduces a constraint on the velicle motion expressing that the velocity of the center of the rear axle is colinear with the orientation axis of the vehicle base. This constraint is of a non-holonomic type, i.e., it is not integral,le, and does not allow a closed-form analytical resolution for the vehicle trajectory.

Only a few authors (e.g., see Refs. 1-6) have studied the problem of generating trajectories for mobile robots witl non-holonomic constraints. These authors have traditionally followed two main types of approaches. In the first type, geometric reasoning is utilized to construct feasible trajectories by assembling arcs of simple curves. Loss, Boulle, and Tournassoud" proposed a set of trajectories built up with two arcs of tangent circles providing adequate trajectories for medium range goal, however generating overly long trajectories for close goals or very distant goals. Kanayama and Hartman ${ }^{2}$ have used clothoid curves to design trajectories, the drawbacks of which also are the lengthy paths with no use of the reverse motion capability, and the complicated geometric computations when steering angle limits are taken into account and distances to obstacle are to be calculated. In the second type of approach, paths are generated by searching the configuration space of the robot, applying the non-holonomic constraint as an additional heuristic at every step of the search. Barraquand and Latombe ${ }^{3}$ proposed an elegant method along these lines based on hierarchial bit-map discretization and potential field functions. The method is generalizable to robots with large numbers of degrees of freedom, however is accurate only to within a mesh spacing, necessitate significant memory requirement and computation time, and is limited to bounded domains since search in configuration space is necessary.

The method proposed here is following the first type of approach, based on geometric reasoning, and remedies some of the drawbacks mentioned above by generating deterministic trajectories between all pairs of configurations (position $x, y$ and orientation $\Theta$ ) of the robot, providing length-optimum paths between distant configurations while utilizing the forward and reverse modes of motion of the vehicle to allow complex naneuvering between close locations with large changes in vehicle oricntation.

\section{Kinematics of a Car-Like Mobile Robot}

We will call "car-like nobile robot" a front-wliecl-drive fourwheel vehicle (see Fig. 1). Let us denote by $L$ the distance between the 2 axes of the wheels, by $\theta$ the angle between the major axis of the robot and the $x$ axis of the alosolute reference frame, by $\phi$ the steering angle (i.e. the orjentation of the fromt wheels with resject to the major axis of the robot), by $x_{M}, y_{M}$, and $V_{M}$ the coordinates and the velocity of $M$, the midelle of the axle of the rear wheels.

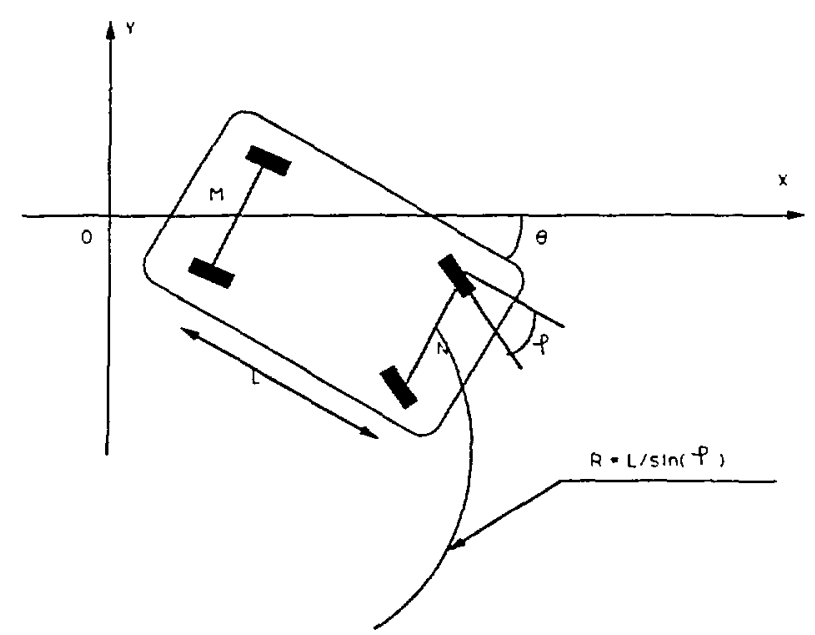

Fig. 1. Scheinatic of a car-like robot.

We assune that there is no slipping of the rear wherls, therefore the velocity vector of $M$ is always colinear with the major axis of the robot:

$$
\frac{d x_{M}}{d t} * \sin \theta-\frac{d y_{M}}{d t} * \cos \theta=0
$$

This equation is not integrable. It is a constraint on the velocity of the robot but does not affect the dimension of the space of configurations (the vehicle can have any orientation at any position). On the other hand, for a given configuration, the space of achicvable velocities has a dimension of only two (the velocity must have the same orientation as the roloot). This is therefore a non-holononic constraint (e.g., sec Refs. 3, 5, 6, 7,8 ).

Another characteristic of the car-like robots is that the steering angle is limited:

$$
|\phi| \leq \phi_{\max }<\frac{\pi}{2}
$$

Let us denote by $N$ the niddlc of the front axle. Let $x_{N}$ and $y_{N}$ be the coorclinates, and $V_{N}$ the velocity of $N$. Since we assume no slipping of the front wheels: 


$$
\overrightarrow{r_{. v}}=\left(\begin{array}{l}
V_{N} * \cos (\theta+\phi) \\
V_{N} * \sin (\theta+\phi)
\end{array}\right)
$$

Since

$$
\overrightarrow{O N}=\overrightarrow{O M}+\overrightarrow{M N}
$$

we have:

$$
\vec{V}_{N}=\vec{V}_{M} \dot{i} \frac{d \overrightarrow{M N}}{d t}
$$

Hence:

$$
\left\{\begin{array}{l}
V_{N} * \cos (\theta+\phi)=\dot{x}_{M}-\dot{\theta} * L * \sin \theta \\
V_{N} * \sin (\theta+\phi)=\dot{y}_{M}+\dot{\theta} * L * \cos \theta
\end{array}\right.
$$

Combining these 2 equations with the non-holonomic constraint, we obtain:

$$
L \dot{\theta}=V_{N} \sin \phi
$$

which can be rewritten as:

$$
\frac{d s}{d \theta}=\frac{L}{\sin \phi}
$$

where $s$ is the curvilinear distance along the trajectory. The previous equation provides $R$, the radius of curvature of the trajectory according to the steering angle. Given the constraint on the steering angle $\phi_{\text {max }}$, we have an expression for the minimum radius of turn of the vehicle:

$$
|R| \geq R_{\min }=\frac{L}{\sin \phi_{\max }}
$$

\section{Principle of the Algorithm}

A mobile robot moving in a 2-D world has 3 degrees of freedom: the coordinates of a given point of the robot, and the orientation of the robot itself. When path planning is part of a task to be performed by the robot, the 3 degrees of freedom must be taken into account. Picking up a pallet with a forklift or docking at a workstation for manipulation tasks, requires the robot to be at a given point, with a given orientation. Therefore a path is a curve joining 2 points and providing a change in the robot orientation.

Changing the orientation of the vehicle can be done only when the vehicle is moving. To change the orientation $\theta$ of the vehicle with a minimum length of trajectory, we need to maximize $\frac{d \theta}{d \theta}$, namely the curvature of the trajectory. This is obviously achieved when the vehicle is moving along a circle the radius of which is the minimum radius of curvature. The problem is that circles take a heavy toll on the length of the trajectory: for a long trajectory, a straight line segruent is really needed. Hence the idea of combining arcs of circles and straight line segments.

The constraint imposing a radius of curvature greater than or equal to $R_{\min }$ restricts the shape of the extremities of the trajectory: to reach a given configuration, "the end" of the trajectory must avoid the inside of the two circles on the sides of the final position of the robot (see Fig. 2). More precisely, 2 such circles can be associated to both the initial configuration and the final configuration of the trajectory. If the robot enters either of the circles related to the final configuration, it will have to get out of it to reach the final configuration. Similarly, from the initial configuration, in order to enter one of the circles, the robot must go away from them first. Therefore the trajectories are designed the following way: the robot moves along one of the circles related to the initial configuration in order to be oriented towards the goal, no matter what the final orientation must be. Then, it moves along a straight line segment in the direction of the goal. The last part of the trajectory is meant to provide the desired final orientation: the robot moves along one of the circles related to the final configuration. To be physically achievable, the trajectory must provide a continuous orientation to the robot. Therefore, the straight line segment must be tangential to the circles (see Fig. 3).

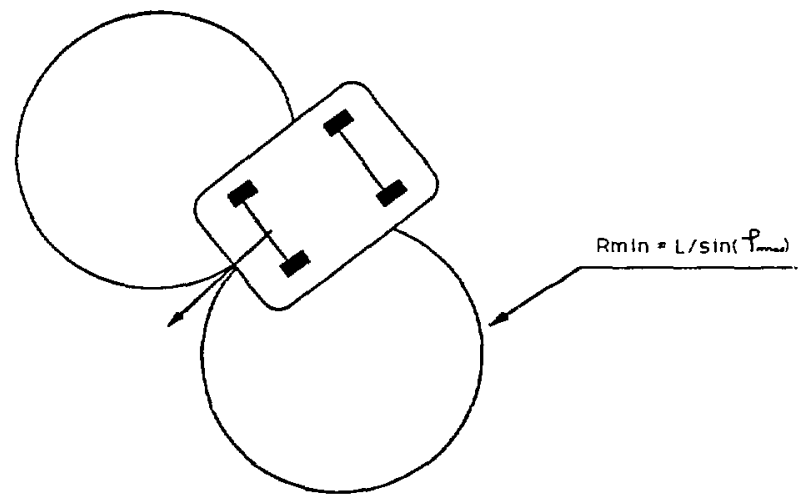

Fig. 2. Minimum radius circles related to a given configuration.

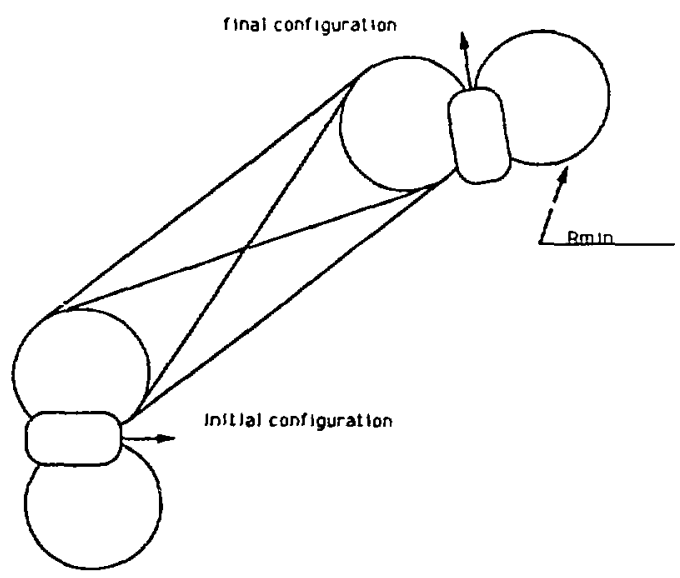

Fig. 3. Possible paths joining two circles.

If the distance between the center of 2 circles is greater than the sum of their 2 radii, there are 4 common tangents to the circles. Since there are 4 possible couples of circles, there are 16 possible paths. The 16 paths provide the right orientation modulo $\pi$. Only 8 provide the desired orientation, the otliers lead to the opposite. Among the 8 remaining paths, the preferred trajectory is selected using a criterion, e.g., shortest length as shown in Fig. 4.

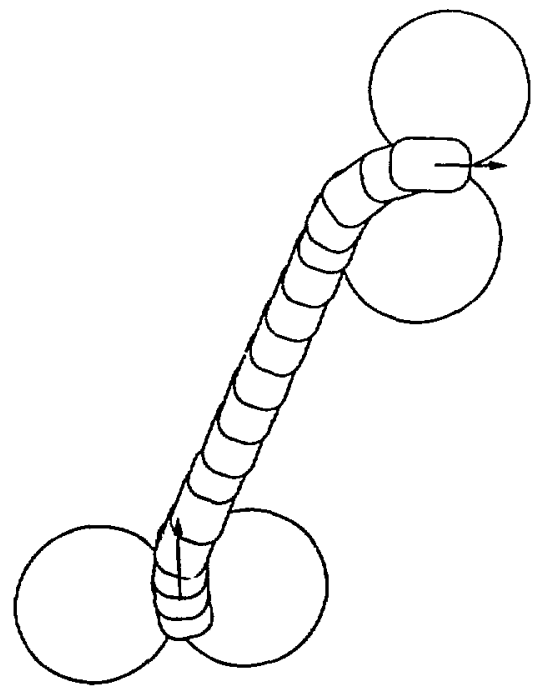

Fig. 4. Example of trajectory joining two configurations. 
If the distance between the 2 chosen circles is smaller thau $2^{*} R_{\min }$, the circles have only 2 common tangents but the principle reriains the same.

When the initial and final positions of the robot are very near each other, backing up ard maneuvering are often involved. The method provides the maneuvers in a straight forward manner (e.g., see Figs. 5 and 6).

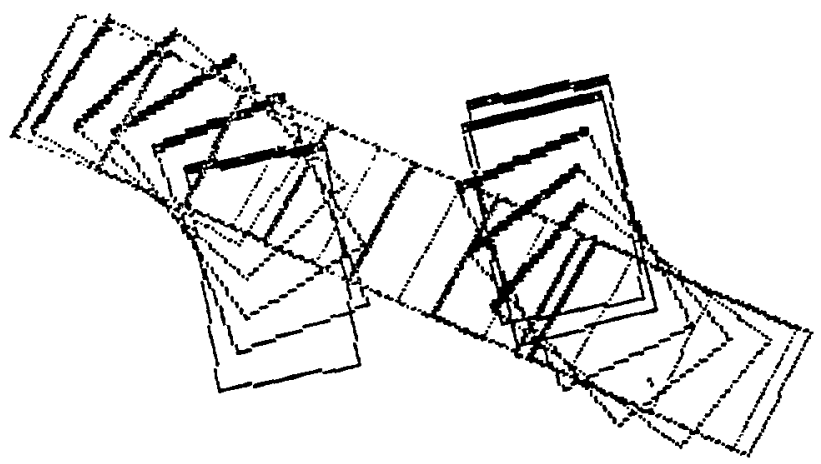

Fig. 5. Eximple of maneuvering.

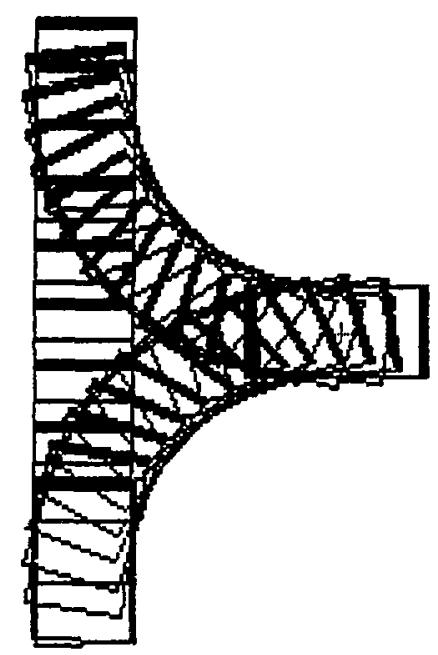

Fig. 6. Example of maneuvering to reverse the dircetion of motion.

This method provides a continuous orientation for the robot because the circles and the segment are tangent. However, the curvature of the obtained curve is discontinuous: it is a non-zero constant on the circles and zero on the straight line segments. From a mechanical point of view, it means that the orientation of the wheels must change suddenly at one point. This does not mean that the trajectory is not achievable, it only means that since the robot cannot turn its wheels instantaneously, it has to stop to do so, and resume moving when the steering angle is correct. This already takes place in cases where the robot has to reverse its velocity to maneuver, which is a common occurrence in an obstructed environment. For the other casis, we describe below a method to provide continuous curvatures along the trajectories.

\section{Taking Into Account the Steering Rale}

In this section, the hypotheses about the robot are t.je same as in the previous section except that there is an additional constraint concerning the stecring angle:

$$
|\dot{\phi}| \leq \dot{\phi}_{\max }
$$

Let us denote by $f_{c}$ the centrifugal force acting on the robot. Experimentally, one can establish that there is a maximum centrifugal force $f_{c \max }$ beyond which the robot caunot be controlled properly. If $\rho$ is the radius of curvature of the trajectory, $V$ the velocity of the robot, and $M$ its mass, we have:

$$
f_{\mathrm{c}}=\frac{M+V^{2}}{\rho}
$$

Since

$$
f_{c} \leq f_{c \max }
$$

We have:

$$
V \leq \sqrt{\frac{\rho * f_{\mathrm{cmax}}}{M}}
$$

A car-like robot is usually a large, heavy vehicle. When the robot is moving along a circle, the velocity must therefore be fairly low. Let us assume that the stecring angle and the velocity of the robot are varying as:

$$
\left\{\begin{array}{l}
v=V+\gamma * t \\
\phi=\phi_{\max }-\dot{\phi}_{\max } t
\end{array}\right.
$$

then:

$$
f=\frac{d s}{d \theta}=\frac{L}{\sin \phi}
$$

Since

$$
d s=(V+\gamma t) d t
$$

we have:

$$
d \theta=\frac{1}{L} *(V+\gamma t) \sin \left(\phi_{\max }-\dot{\phi}_{\max } t\right) d t
$$

If we denote by $T$ the tine needed to change the steering angle from $\phi_{\max }$ to 0 , (i.e., cliange from motion along the circle to motion along a straight line) the corresponding variation of the robot orientation is:

$$
\Delta \theta=\int_{0}^{T} d \theta
$$

and the equations of the trajectory are:

$$
\left\{\begin{array}{l}
x_{N}(s)=\int_{0}^{s} \cos \theta d s \\
y_{N}(s)=\int_{0}^{s} \sin \theta d s
\end{array}\right.
$$

Figure 7 shows the curve corresponding to this transition. Similar transition trajectories can be calculated for a varicty of steering rates and velocity constraints other than those expressed in Eq. (14) and shown in Fig. 7. The interesting point is that since the robot moves at a constant velocity on the circle, the same transition trajectory can be utilized to plan the rolot path from any point on the circle when clanging its stecring angle from $\phi_{\max }$ to 0 . However complicated the transition trajectory equations may be, they can be solved off-line and the computed trajectory can be stored in the robot memory. In order to find the path requiring a continuous steering angle, the problem then reduces to finding the straight line segment intersecting 2 circles with a given angle (always the same). An example of such a full trajectory is shown in Fig. 8 . 


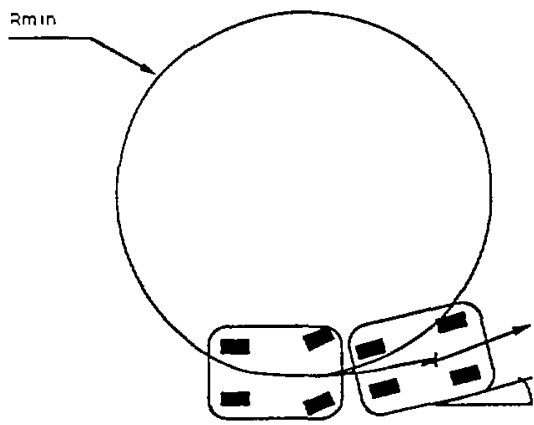

Fig. 7. Transition trajectory for a linear variation of the steering angle.

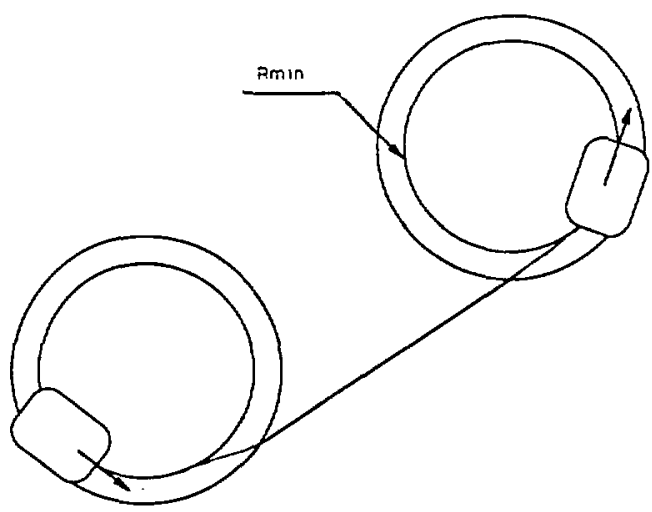

Fig. 8. Example of trajectory taking into account the nonholonomic, steering limit and steering rate constraints.

\section{Composite Trajectories in Obstructed Environments}

In environments where few or no obstacles exist, the method presented in the previous sections is particularly efficient since at least 4 , and in most cases 8 alternative trajectories are generated for each pair of initial and final configurations. If some of these paths are not feasible because of the presence of an obstacle, the alternative paths can be directly exannined for suitability. For example, if an obstacle existed in the upper left-space or brtween the initial and final configuration of the case slown in Fig. 5 , the alternative trajectory shown in Fig. 9 (which was generated at the same time as that of Fig. 5) could be used.

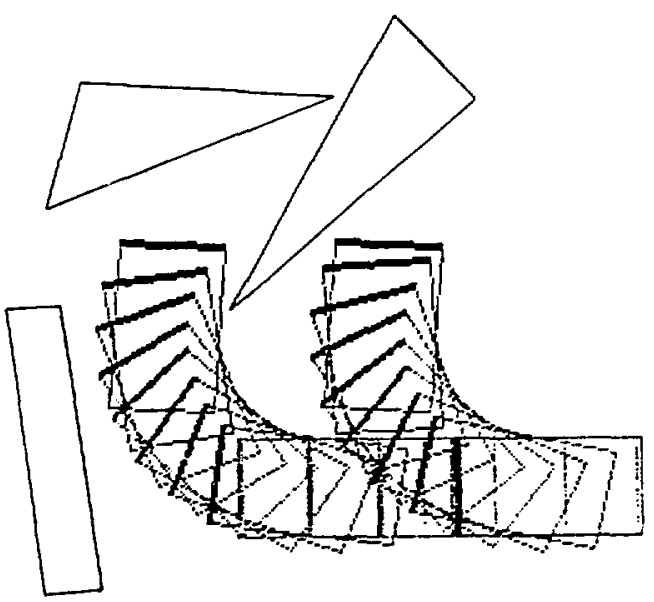

Fig. 9. One of the available alternate trajectories if obstacles obstruct the trajectory shown in Fig. 5.

In cases when none of the basic trajectories provide collisionfree motion, subgoaling can be utilized. Two methods, dependirg on whether the algorithm is used for a teleoperated or an autonomous system, have been investigated. In the former casc, the operator specifies on the man-machine interface systen the location of potential intermediate configuration $\mathrm{gonls}$, which the trajectory generation module interactively examines until an appropriate set of subgoals has been found in between which collision-free trajectories exist. Figure 10 is a copy of thre display screen during such an interactive session juvolving a forklift vehicle. ${ }^{9}$ The configuration subgoals are identified by the numbered arrows.

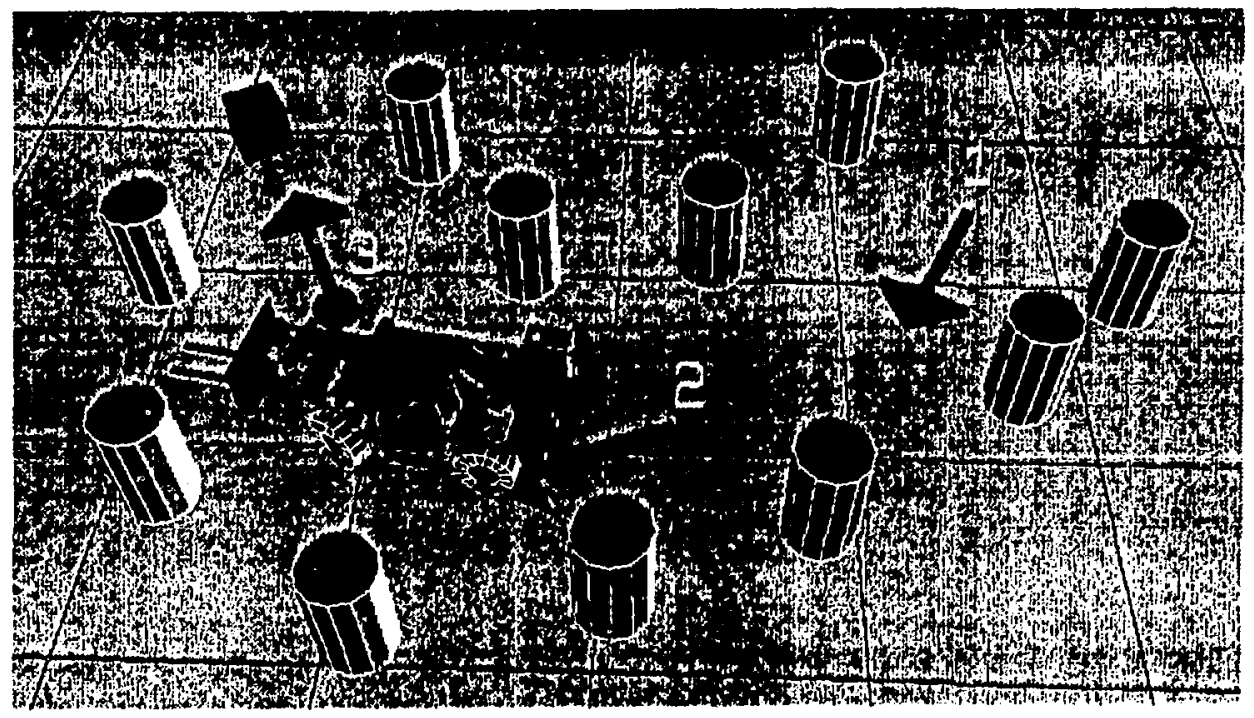

Fig. 10. Copy of an operator-machine interface screcn display during an interactive subgoal sclection session. 
In the second method, used for fully autonomous systems, the basic trajectory generation module calls upon a navigation planner to autonomously generate the set of configuration subgoals that lead to collision-free motion (see Ref. 10). The subgoal selection can be performed globally if the environment is a priori fully known, or locally, based on sensor data, for a priori unknown or dynamic environments. Once the set of subgoals has been established, the trajectory planning module described in the previous sections generates the trajectories joining the configuration subgoals. Figure 11 shows an exanple of such generation of a composite trajectory for a mobile robot having the task to reach the configuration labeled 3 on the figure from the configuration labeled 0 , in a highly obstructed environment. The configuration subgoals are represented by the arrows labeled 1 and 2 , and the calculated composite trajectory involving several "maneuvers" is shown by the succession of robot configurations along the path.

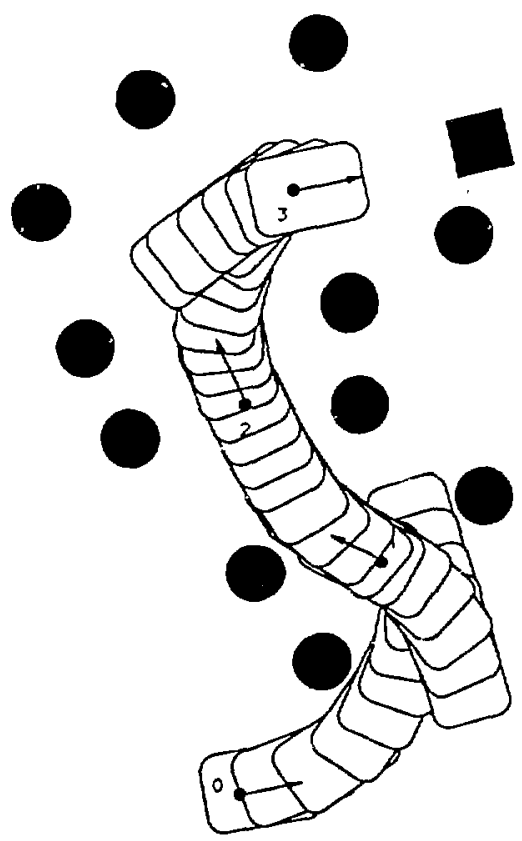

Fig. 11. Example of a composite trajectory involving subgoals in a highly obstructed environment.

\section{Conclusion}

A methodology and algorithm for generating trajectories for mobile robots with non-holonomic, steering angle and steering rate constraints has been presented. The main advantage of the method is that it is deterministic and provides trajectories joining all pairs of configurations of the robot. The method also takes advantage of the forward and reverse mode of motion of the vehicle as well as combination of these on trajectories requiring maneuvering. The algorithm cas be easily used by an operator who just has to specify the desired final configuration of the vehicle. If the robot is used in an antonomons mode, the method can be used as a busic incremental trajectory generator in coordination with a global navigation planner. The fart that the basic trajectories all have the sane compesition, with wrellknown geometric clements, enables one to carry out easy aud fist computations of the distances with respect to olsstarles. This and the consjederation that only a fow milliseconds of run tinie on a micro Vax are necessary to generate the trajectories, makes the method quite attractive for real-tine navigation and motion planners in cobstructed and/or a priori unkmown entvironuents.

\section{References}

(1) E. Loss, M. Boulle, and P. Tournassoud, "Local Trajectory Generation for a Mobile Robot," INRIA France, Internal Report, 1988.

[2] Y. Kanayarna and B. I. Hartman, "Smootl Local Path Planning for Autonomous Velicles," in Procedings of the 1989 IEEE International Conference on Robotics and Automation, Scottsdale, Ariz., May 14-17, 1989, pp. 1265-1270.

[3] J. Barraquand and J. C. Latombe, "On Nonlolonomic Mobile Robots and Optinin Maneuvering," in Proccedings of the 4th IEEE International Symposium on Intelligent Control, Albany, N.Y., 1989, pp. 1-8.

[4] S. Sastry, Mathematical Questions in Robotics, Aneriran Mathematical Society Short Course Series, Louisville, $\mathrm{Ky}$, Jan. 16-17, 1990.

[5] J. P. Laumont, "Feasible Trajertories for Mobile Robots with Kinematic and Environment Constraints," in Proceedings of the International Conference on Intelligent Autonomous Systems, Amsterdam, The Netlicrlands, Dec. 8-11, 1986, pp. 346-354.

[6] J. P. Laumont, "Finding Collision-Free Smooth Trajectories for a Non-Holonnmic Molsile Rolot," in Procecdings of the 10tl International Joint Conference on Artificial Intelligence, Milano, Italy, Ang. 23-28, 1987, pp. 1120-1123.

[7] A. M. Bloch and N. H. McClamirod, "Control of Mechanical Systems with Classicai Non-Holononic Constraints," in Proceedings of the 28th IEEE Conference on Decision and Control, Tanpa, Fla., Dec. 13-15, 1989, pp. 201-205.

[8] S. Sastry and Z. Li, "Robot Motion Planning with Non-Holonomic Constraints," in Procecdings of the 28th IEEE Conference on Decision and Control, Tampa, Fla., Dec. 13-15, 1989, pp. 211-216.

[9] S. F. Sousk, F. G. Pin, and C. D. Crane, "Motion Plaming for the Universal Self-Deployable Cargo Handler (USDCH)," to appear in Proccedings of the U.S. Army Science Conference, Durham, N.C., June 12-15, 1990.

[10] H. A. Vasseur, F. G. Pin, and J. R. Taylor, "Path Planning and Control for Autonomous Mobile Robots with Nonholonomic, Steering Angle and Sterring Rate Constraints," subnitted to Robotics and Autonomous Systems, 1990. 